\title{
COMPUTER SIMULATION OF THE STRESS-STRAIN STATE OF THE PLATE WITH CIRCULAR HOLE AND FUNCTIONALLY GRADED INCLUSION
}

\author{
Eteri L. Hart*, Bohdan I. Terokhin ${ }^{\dagger}$
}

Communicated by Prof. V.O. Kapustyan

\begin{abstract}
Computer simulation of the behavior of a thin elastic rectangular plate with a circular hole and an annular inclusion made of functionally graded material has been carried out. Using the finite element method, the influence of the geometric and mechanical parameters of the inclusion on the concentration of stresses around the hole is investigated and various laws of the change in the modulus of elasticity of a functionally graded material are specified. A comparative analysis of the results has been carried out. The recommendations for reducing stress concentration are given.
\end{abstract}

Key words: Elastic plate, circular hole, inclusion, functionally graded material, stress concentration factor, finite element method.

2010 Mathematics Subject Classification: 35J50, 35J57, 39B42,74G70,74K20, 74S05.

\section{Introduction}

Plates and shells with holes are quite often used in various fields of technology, power engineering, construction, etc. The presence of holes leads to the appearance of local (additional) stresses, which can be several times higher than the basic stresses in an element that is not weakened by a concentrator. Under certain conditions, this initiates destruction processes. That is why, in order to increase the strength of the structure, it is necessary to look for ways to influence the distribution of stresses in the body, in particular, on the value of the stress concentration factor (SCF). One of these methods is the use of inclusions around the holes, of various geometric shapes and mechanical properties $[5,6,11]$.

Recently, in the manufacture of plate-shell structural elements of new technology, in particular, aerospace, functionally graded materials (FGM) $[16,17]$ are used, which are classified as materials with unique mechanical, technological and special properties. A specific feature of FGM is a smooth change in mechanical properties and chemical composition in a certain direction. The gradient structure of materials provides an increase in the level of service properties of parts and structural elements, taking into account the respective operating conditions. FGMs have

\footnotetext{
*Department of Theoretical and Computer Mechanics, Oles Honchar Dnipro National University, 72, Gagarin av., Dnipro, 49010, Ukraine, hart@ua.fm

${ }^{\dagger}$ Department of Theoretical and Computer Mechanics, Oles Honchar Dnipro National University, 72 , Gagarin av., Dnipro, 49010, Ukraine, bogdan.teryokhin@gmail.com

(C) E. L. Hart, B. I. Terokhin, 2021.
} 
high strength and a set of properties when working for impact, wear, fatigue, they can withstand increased cyclic and alternating loads, etc.

Taking into account the presence of this kind of material inhomogeneity leads to an increase in the complexity of the mathematical model of the problem. Finding the exact solution of the obtained boundary value problem in an analytical form is possible only in some individual cases of the load of bodies and under certain conditions of their fixation [3,12,13,19-23]. Therefore, when studying the stress-strain state (SSS) of structures made of FGM and with various inhomogeneities (holes, inclusions, etc.), it is advisable to use numerical methods of mechanics, which, unlike analytical ones, are quite universal and effective for solving a wide class of problems. [2, 4,6-11]. The most effective are grid methods: the finite element method [27], the finite difference method, the method of local variations and their projection-iterative implementation schemes [6-8], which accelerate the convergence of the process of obtaining a solution to the problem and significantly reduce the cost of computer computation time.

FGM mechanics has attracted great interest in the last two decades, and many works have appeared on theoretical, numerical and experimental studies of FGM. Thus, in [1], analytical solutions of mixed axisymmetric problems for functionally graded media were obtained. In $[25,26]$, using an analytical method, the stress distribution in a plate with an FGM with a circular hole was investigated. In [15], the stress concentration in multi-wedge systems with functionally graded wedges was estimated. In [14], using various isoparametrical finite elements, the SCF was determined in the vicinity of a circular cut in an inhomogeneous plate under uniaxial tension, in [18] the SCF was determined around a circular cut in an FGM plate under biaxial tension and shear.

In this work, using the finite element method (FEM), a computer simulation of the behavior of a thin elastic rectangular plate with a circular hole and an annular inclusion under the action of a uniaxial tensile load was carried out for various properties of the inclusion material and its dimensions.

\section{Statement of the Problem}

A thin elastic homogeneous isotropic plate is given with dimensions $\mathrm{a} \times \mathrm{b}$ and thickness $\mathrm{t}$ with a centrally located circular hole of radius $R$ and an annular inclusion of radius $\mathrm{R}_{1}$ (Fig. 2.1). A uniform uniaxial tensile load $p=$ const acts on the plate, which does not lead to the appearance of plastic deformations.

It is believed that the inclusion is modeled by an insert, which is in the plane of the plate and has the same thickness as it; conditions of rigid adhesion are specified at the boundary of the inclusion with the plate.

FGM inclusions with arbitrary radial elastic properties are considered. In the numerical examples, six model materials were selected with the same Poisson's ratio $\nu_{0}=0.25$, but with different inclusion elastic modulus $E_{i}(r)(i=\overline{1,6})$. The first three materials have the following laws of change in the modulus of elasticity $E_{i}(r)(i=\overline{1,3})$ : 


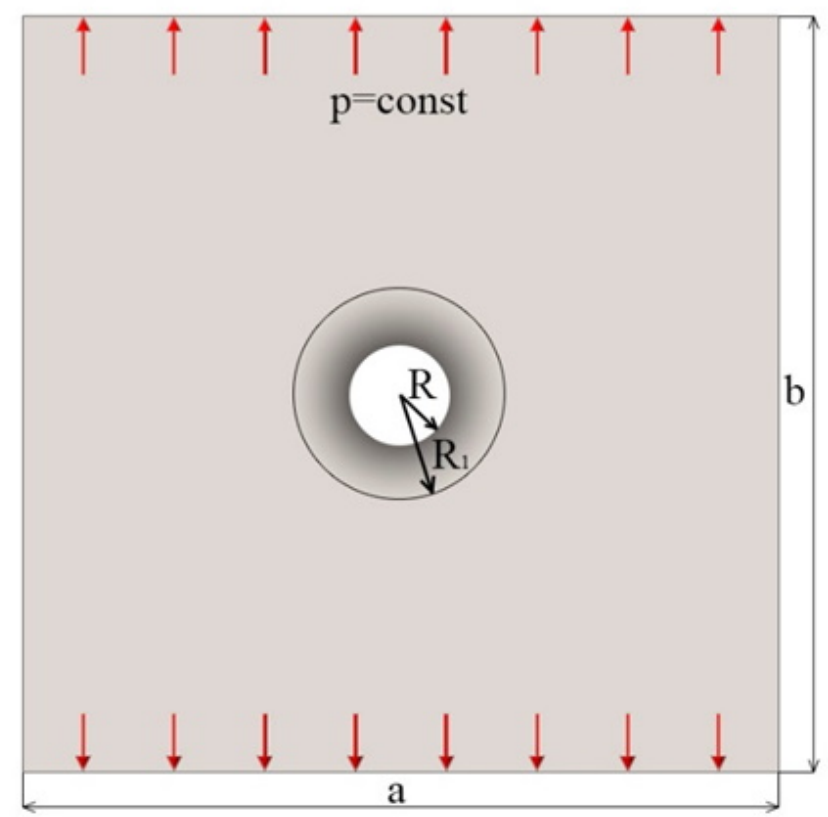

Fig. 2.1. Plate geometry and loading diagram

$$
\begin{gathered}
E_{1}(r)=\left\{\begin{array}{l}
E_{0}(1+l), l \in[0 ; 0,5] \\
E_{0}(2-l), l \in[0,5 ; 1]
\end{array} ;\right. \\
E_{2}(r)=\left\{\begin{array}{l}
E_{0}\left(1+\frac{5}{4} l\right), l \in[0 ; 0,4] \\
1,5 E_{0}, l \in[0,4 ; 0,6] \\
E_{0}\left(2 \frac{1}{4}-\frac{5}{4} l\right), l \in[0,6 ; 1]
\end{array} ;\right. \\
E_{3}(r)=\left\{\begin{array}{l}
E_{0}\left(1+\frac{5}{3} l\right), l \in[0 ; 0,3] \\
1,5 E_{0}, l \in[0,3 ; 0,7] \\
E_{0}\left(2 \frac{2}{3}-\frac{5}{3} l\right), l \in[0,7 ; 1]
\end{array}\right.
\end{gathered}
$$

where $E_{0}=100 \mathrm{GPa}$ is the modulus of elasticity of the plate; $0 \leqslant l \leqslant 1$ is the normalized parametric distance in the radial direction from the center of the hole along the width of the inclusion $h=R_{1}-R: l=(r-R) /\left(R_{1}-R\right), r$ is the distance from the center of the hole to an arbitrary point of inclusion; $R$ and $R_{1}$ are the radii of the hole and the annular inclusion, respectively.

Note that for the given materials of inclusions (2.1)-(2.3), the values of the elastic modulus are in the range from 100 to $150 \mathrm{GPa}$. For three other model materials, the laws of change in the modulus of elasticity $E_{i}(r)(i=\overline{4,6})$ are similar, but the values of the modulus of elasticity vary in the range from 100 to $200 \mathrm{GPa}$. 
In Fig. 2.2 we show a graphical representation of the laws of change in the elastic modulus of an FGM inclusion. Lines 1-3 correspond to dependencies (2.1)(2.3).

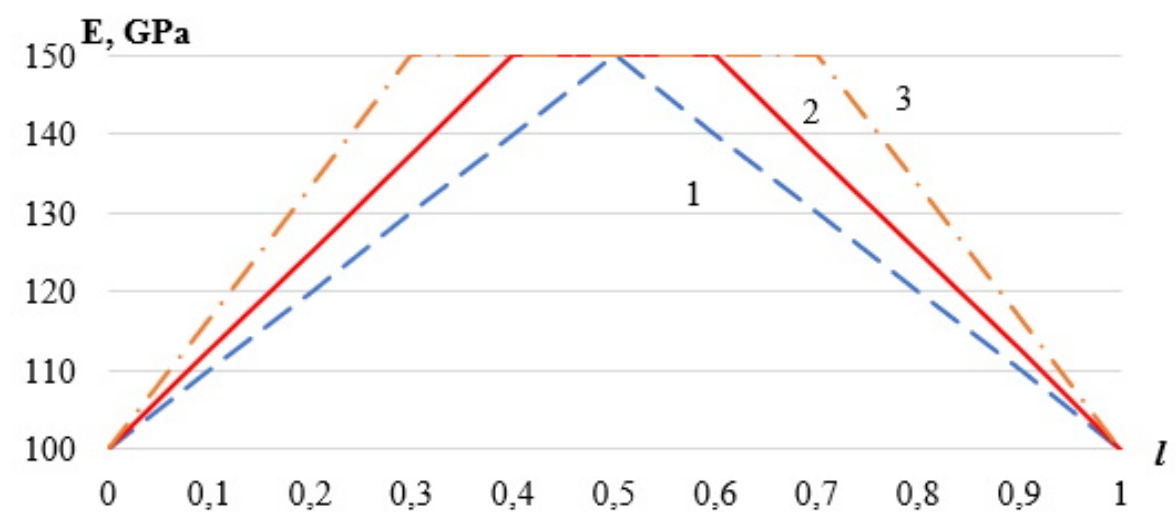

Fig. 2.2. The laws of change in the elastic modulus of FGM inclusions

It is necessary to determine the SSS of a given plate for each of the specified variants of inclusions to study the effect of the size and nature of the change in the modulus of elasticity of the inclusion on the SSS of the plate in the zones of local stress concentration and the effect on the value of the SCF; to carry out a comparative analysis of the results for a plate without an inclusion with a plate in the presence of an FGM-inclusion (Fig. 2.2).

\section{Mathematical Model of the Problem}

The relationship of the theory of elasticity for the region of a plane-stressed plate has the form $[23,24]$ :

- equilibrium equations:

$$
\left\{\begin{array}{l}
\frac{\partial \sigma_{11}}{\partial x}+\frac{\partial \sigma_{12}}{\partial y}+R_{1}=0 \\
\frac{\partial \sigma_{21}}{\partial x}+\frac{\partial \sigma_{22}}{\partial y}+R_{2}=0
\end{array}\right.
$$

or in matrix form

$$
[\partial]^{T}\{\sigma\}+\{R\}=0
$$

where $\left\{\begin{array}{l}R_{1} \\ R_{2}\end{array}\right\}$ is a vector of volumetric forces, $[\partial]=\left[\begin{array}{ll}\partial / \partial x & 0 \\ 0 & \partial / \partial y \\ \partial / \partial y & \partial / \partial x\end{array}\right]$ is the differentiation matrix, $\{\sigma\}=\left\{\begin{array}{l}\sigma_{11} \\ \sigma_{22} \\ \sigma_{12}\end{array}\right\}$ is the stress vector $\left(\sigma_{12}=\sigma_{21}\right)$; 
- geometric equations (in Cauchy form):

$$
\varepsilon_{11}=\frac{\partial u_{1}}{\partial x}, \quad \varepsilon_{22}=\frac{\partial u_{2}}{\partial y}, \quad \gamma_{12}=\frac{\partial u_{1}}{\partial y}+\frac{\partial u_{2}}{\partial x}
$$

or in matrix form

$$
\{\varepsilon\}=[\partial]\{u\}
$$

where $\{u\}=\left\{\begin{array}{l}u_{1}(x, y) \\ u_{2}(x, y)\end{array}\right\}$ is the displacement vector, $\{\varepsilon\}=\left\{\begin{array}{l}\varepsilon_{11} \\ \varepsilon_{22} \\ \gamma_{12}\end{array}\right\}$ is the deformation vector;

- physical equations:

$$
\varepsilon_{11}=\frac{\partial u_{1}}{\partial x}, \quad \varepsilon_{22}=\frac{\partial u_{2}}{\partial y}, \quad \gamma_{12}=\frac{\partial u_{1}}{\partial y}+\frac{\partial u_{2}}{\partial x}
$$

which with respect to stresses have the form:

$$
\left\{\begin{array}{l}
\sigma_{11}=\frac{E}{1-\nu^{2}} \varepsilon_{11}+\frac{E \nu}{1-\nu^{2}} \varepsilon_{22}, \quad \sigma_{12}=\gamma_{12} \frac{E}{2(1+\nu)}, \\
\sigma_{22}=\frac{E \nu}{1-\nu^{2}} \varepsilon_{11}+\frac{E}{1-\nu^{2}} \varepsilon_{22},
\end{array}\right.
$$

in the matrix form $\{\sigma\}=[E]\{\varepsilon\}$, where $[E]$ is the elastic matrix, $\nu$ is the Poisson's ratio,

$$
[E]=\left[\begin{array}{ccc}
\frac{E}{1-\nu^{2}} & \frac{E \nu}{1-\nu^{2}} & 0 \\
\frac{E \nu}{1-\nu^{2}} & \frac{E}{1-\nu^{2}} & 0 \\
0 & 0 & \frac{E}{2(1-\nu)}
\end{array}\right], \quad\{\varepsilon\}=\left\{\begin{array}{c}
\frac{\partial u_{1}}{\partial x} \\
\frac{\partial u_{2}}{\partial y} \\
\frac{\partial u_{1}}{\partial y}+\frac{\partial u_{2}}{\partial x}
\end{array}\right\}
$$

The work of internal forces on possible displacements:

$$
\delta U=\frac{1}{2} \int_{\Omega}\left(\sigma_{11} \delta \varepsilon_{11}+\sigma_{22} \delta \varepsilon_{22}+\sigma_{12} \delta \gamma_{12}\right) d \Omega=\frac{1}{2} \int_{\Omega}\{\delta \varepsilon\}^{T}\{\sigma\} d \Omega .
$$

The work of external forces on possible displacements:

$$
\delta A=\int_{L}\{P\}^{T}\{\delta u\} d L
$$

where $\{P\}=\left\{\begin{array}{l}p_{x}(x, y) \\ p_{y}(x, y)\end{array}\right\}$ is the external load vector.

In the case of a uniform uniaxial tensile load $p_{x}(x, y)=p=$ const; $p_{y}(x, y)=$ $=0$.

For FGM in formulas (3.3)-(3.5), we set $E=E(x, y)$.

The functional of the total potential energy of deformation of a plate, which is loaded in its plane, has the form: 


$$
\prod=\frac{1}{2} \int_{\Omega}\{\delta \varepsilon\}^{T}\{\sigma\} d \Omega-\int_{L}\{P\}^{T}\{\delta u\} d L
$$

\section{Solution Method}

The solution of the obtained variational problem was carried out using the FEM [27]. The main idea of this method when analyzing the behavior of a structure is as follows: a continuous medium (the structure as a whole) is modeled by dividing it into subdomains (finite elements), in each of which the behavior of the medium is described using a separate set of selected, so-called basis functions representing stresses and move in the specified area. Within each finite element, the selected continuous function is approximated by a polynomial of some degree. As a result, the original variational problem is replaced by a discrete model, a system of linear or nonlinear algebraic equations with unknown values of the sought function at the nodes of the finite element mesh.

The calculations were carried out using triangular (six-node) Lagrangian finite elements of the second degree (Fig. 4.3, a), while the unknown displacement functions inside each finite element are approximated by a quadratic polynomial. In the areas of stress concentration, an adaptive mesh with a refinement factor of 10 was used (Fig. 4.3, b).

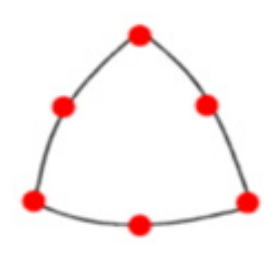

a)

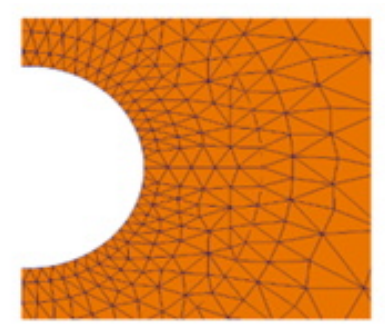

b)

Fig. 4.3. Breakdown of an area into finite elements: a) the type of the final element; b) a fragment of an adaptive finite element mesh

\section{Numerical Analysis}

The calculations were carried out on a PC ARTLINE Gaming X75 (X75v16), with an Intel Core i7-10700F processor with a clock rate of $2.9-4.8 \mathrm{GHz}, 32 \mathrm{~GB}$ of RAM, an nVidia GeForce RTX 2060 SUPER video card, system bit width x64. The number of finite elements is 1871 , the number of nodes is 3885 The calculation time on average is $4 \mathrm{~s}$.

Numerical studies were carried out for square plates of thickness $t=0.01 \mathrm{~m}$, with sides $a=b=0.2 \mathrm{~m}$. The radius of the circular hole is $R=a / 20$, the tensile load is $p=10 \mathrm{MPa}$. 
For the purpose of comparative analysis, a calculation was carried out for a homogeneous plate with a circular hole without inclusion. Received $S C F=3.05$; the maximum values of the intensity of deformations in this case, which is in good agreement with the results from [19].

As a result of the computational experiments using the FEM, the distribution of the stress and strain intensities in the plate was obtained, the SCF was calculated for uniaxial tension of the plate with inclusions from the FGM with the inclusion width $\mathrm{R}$ and $2 R$.

When using FGM-inclusions 1, 2, 3 with the width of the inclusion $h=R$, the SCF almost does not change. Calculations for these inclusions at $h=2 R$ are given in Table 1. Here, $\delta_{1}$ and $\delta_{2}$ are the deviation of SCF and the maximum

\begin{tabular}{|c|c|c|c|c|}
\hline Problem & $\mathrm{SCF}$ & $\delta_{1}, \%$ & $\varepsilon_{i}^{\max }, 10^{-4}$ & $\delta_{2}, \%$ \\
\hline FGM inclusion 1 & 2,84 & $-6,9$ & 1,93 & $-9,4$ \\
\hline FGM inclusion 2 & 2,81 & $-7,9$ & 1,89 & $-11,3$ \\
\hline FGM inclusion 3 & 2,79 & $-8,5$ & 1,85 & $-13,1$ \\
\hline
\end{tabular}

Table 1. Stress concentration factor and corresponding deformations in a plate with FGMinclusion at $h=2 R$

value of the intensity of deformation $\varepsilon_{i}^{\max }$ from the corresponding value for the plate without inclusion.

From Table. 1 it can be seen that in the case of an annular inclusion of width $2 R$ from an FGM, the maximum deformations and SCF in a plate with a hole are less than in a plate without inclusions. The smallest SCFs and strains were obtained in the case of FGM inclusion 3.

In the presence of inclusions from FGM of width $2 \mathrm{R}$, a redistribution of stresses occurs along the section $\mathrm{AB}$ from the edge of the inclusion to its middle part. Thus, the maximum stresses decrease, but the stresses increase along the width of the inclusion in the section $\mathrm{AB}$ in the interval $l \in[0,1 ; 0,7]$. The nature of the stress distribution is close to the parabolic pattern (Fig. 5.4).

The SCF and deformations under uniaxial tension of a plate with FGM inclusions 4, 5, 6 with the width of the inclusion $R$ and $2 R$ are shown in Table 2 and Table 3 respectively.

In the case with the inclusion width $h=R$ for all three variants of inclusions, the SCF decreased by about $8 \%$, and the maximum deformations by $13-17 \%$ compared to the plate without inclusions. FGM-inclusion 6 turned out to be the best from the point of view of reducing the concentration of SSS parameters (Fig. $5.5)$. 


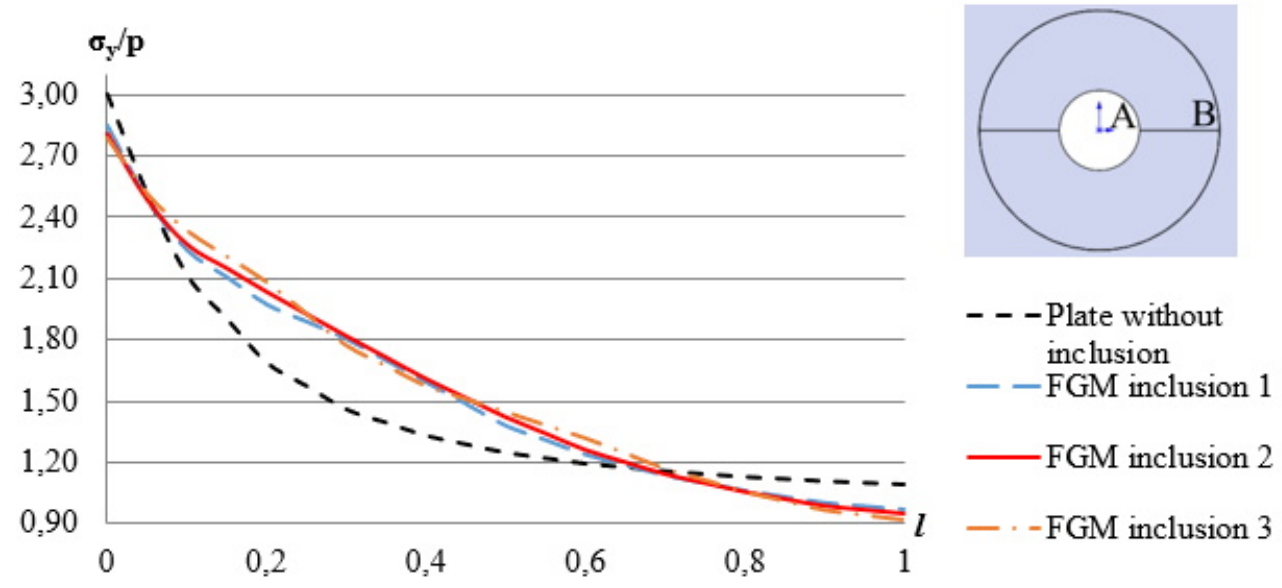

Fig. 5.4. Distribution of relative stresses $\sigma_{y} / p$ in a plate with an FGM-inclusion over the width of the inclusion in the section $\mathrm{AB}$ at $h=2 R$

\begin{tabular}{|c|c|c|c|c|}
\hline Problem & SCF & $\delta_{1}, \%$ & $\varepsilon_{i}^{\max }, 10^{-4}$ & $\delta_{2}, \%$ \\
\hline FGM inclusion 1 & 2,82 & $-7,5$ & 1,85 & $-13,1$ \\
\hline FGM inclusion 2 & 2,79 & $-7,5$ & 1,80 & $-15,5$ \\
\hline FGM inclusion 3 & 2,79 & $-8,5$ & 1,76 & $-17,4$ \\
\hline
\end{tabular}

Table 2. Stress concentration factor and corresponding deformations in a plate with FGMinclusion at $h=R$

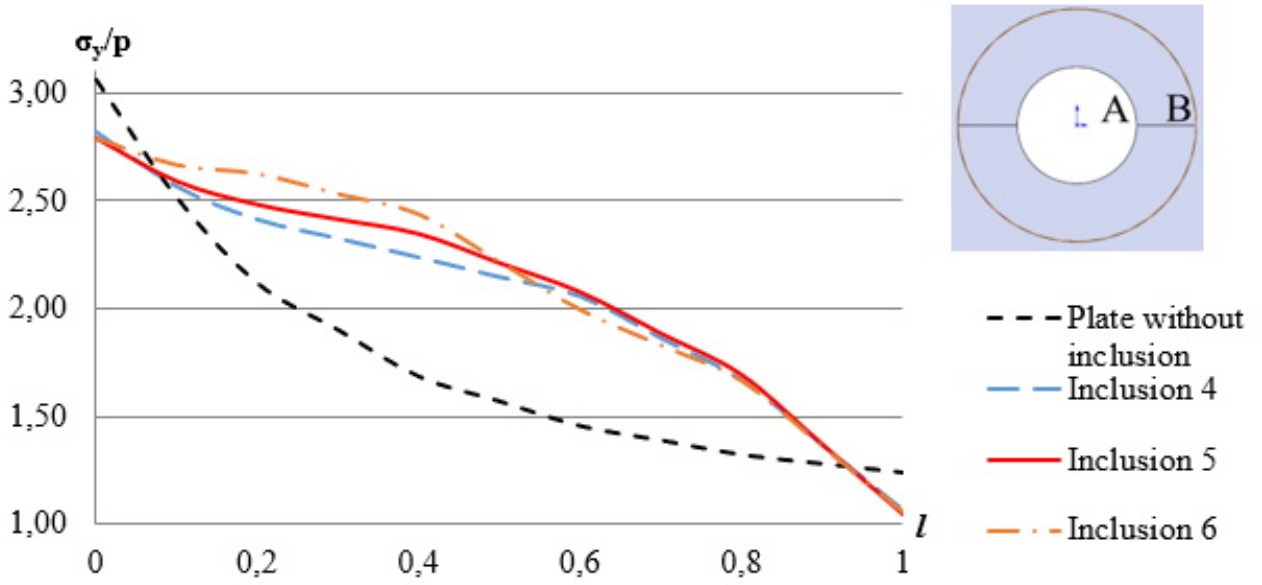

Fig. 5.5. Distribution of relative stresses $\sigma_{y} / p$ in a plate with an FGM-inclusion over the width of the inclusion in the section $\mathrm{AB}$ at $h=R$ 
Analogical calculations were carried out for a plate with an inclusion width equal to $2 R$. The results are shown in Table 3 .

\begin{tabular}{|c|c|c|c|c|}
\hline Problem & SCF & $\delta_{1}, \%$ & $\varepsilon_{i}^{\max }, 10^{-4}$ & $\delta_{2}, \%$ \\
\hline FGM inclusion 1 & 2,67 & $-12,8$ & 1,76 & $-17,4$ \\
\hline FGM inclusion 2 & 2,62 & $-14,4$ & 1,70 & $-20,2$ \\
\hline FGM inclusion 3 & 2,59 & $-15,4$ & 1,64 & $-23,0$ \\
\hline
\end{tabular}

Table 3. Stress concentration factor and corresponding deformations in a plate with FGM inclusion at $h=2 R$

Here, the SCF and the maximum deformations in the plate in the presence of FGM inclusions also turn out to be less than in the case of a plate without inclusions, and the stress across the width of the inclusion in the section $\mathrm{AB}$ increases in the interval $l \in[0,1 ; 0,7]$ (Fig. 5.6). The nature of stress distribution in section $\mathrm{AB}$ for all three inclusions is similar. The best turns out to be FGMinclusion 6 , which makes it possible to reduce the stress concentration by $\sim 15 \%$, and deformations by $\sim 23 \%$.

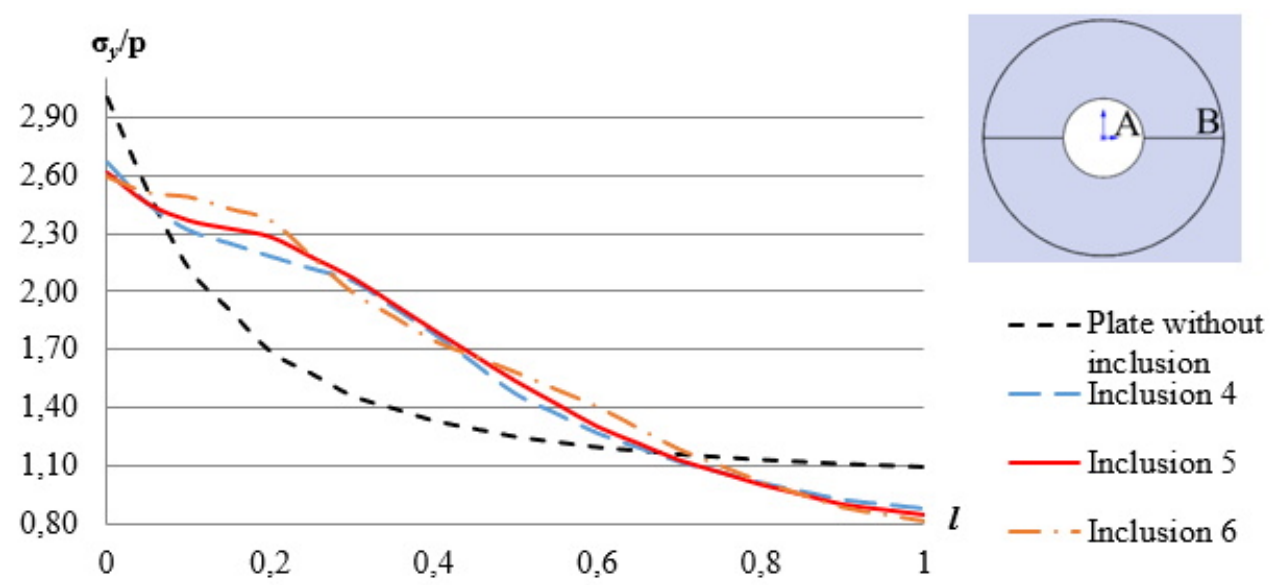

Fig. 5.6. Distribution of relative stresses $\sigma_{y} / p$ in a plate with an FGM-inclusion over the width of the inclusion in the section $\mathrm{AB}$ at $h=2 R$

As an example (see Fig. 5.7), we show the distribution patterns of stress and strain intensities in a plate with FGM-inclusion 1. 


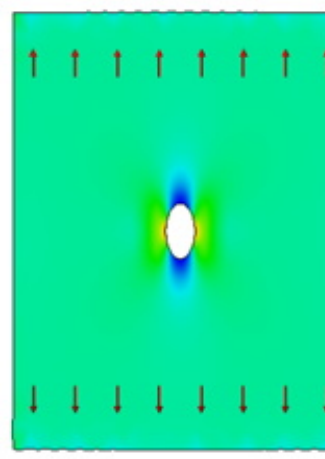

a)

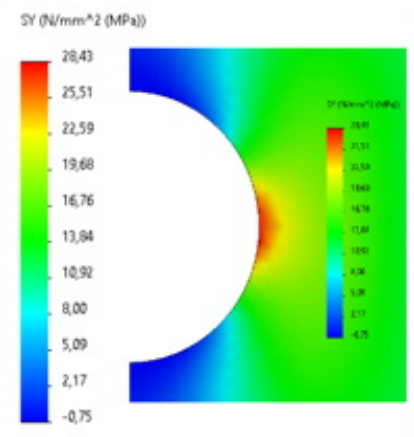

b)

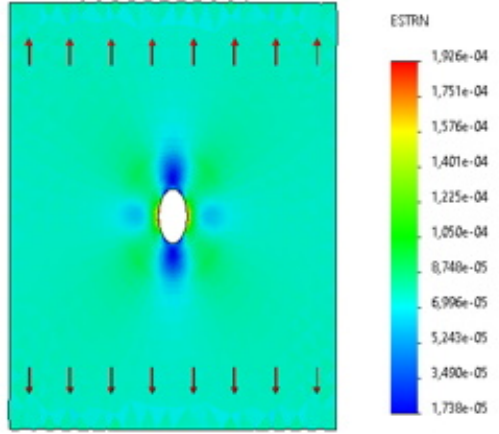

c)

Fig. 5.7. SSS components in a plate with FGM-inclusion 1 at $h=2 R$ :

a) stress intensity; b) the intensity of stresses in the vicinity of the hole; c) the intensity of deformations

\section{Conclusions}

As a result of the computer simulation and numerical study of the effect of a change in the elastic modulus of an inclusion in the radial direction on the distribution of stress and strain intensities in a thin homogeneous plate near a circular hole, it has been established that in the presence of FGM inclusions with certain mechanical properties, it becomes possible to influence not only the SCF value in plate near local stress concentrators, but also on the stress distribution over the width of the inclusion. In a comparative analysis of the results obtained for a plate with an FGM inclusion and a plate without an inclusion, it was shown that the use of FGM inclusions is effective. This makes it possible to simultaneously reduce the concentration of SSS parameters (stresses and strains) around the hole and increase the strength of the plate as a whole.

The nature of the stress distribution in the plate is influenced by both the width of the FGM inclusion and the law of change in the modulus of elasticity: the larger the width of the inclusion and the larger the region with the maximum value of the elastic modulus of the inclusion, the greater the effect of the inclusion on the value of SCF and the magnitude of maximum deformations. The influence of the range of the change in the value of the elastic modulus is also established: the greater it is, the greater the effect on the value of the SCF in the plate.

\section{References}

1. S.M. Aizikovich, V.M. Aleksandrov at al. Analytical solutions of mixed axisymmetric problems for functionally graded media, Fizmatlit, Moscow, 2011.

2. S.K. DeB NAth, C.H. Wong, S.-G. Kim, A finite-difference solution of boron/epoxy composite plate with an internal hole subjected to uniform tension/displacements using displacement potential approach, Intern. J. Mech. Sci., 58 (2012), 1-12. 
3. X.Q. FAnG, C. Hu, S.Y. Du, Strain energy density of a circular cavity buried in semi-infinite functionally graded materials subjected to shear waves, Theoret. Appl. Fracture Mech., 46(2006), 166-174.

4. V.S. Gudramovich, É.L. Gart, K.A. Strunin, Modeling of the behavior of plane-deformable elastic media with elongated elliptic and rectangular inclusion, Materials Science, 52 (6) (2017), 768-774.

5. E.L. Hart, V.S. Hudramovich, Projection-iterative schemes for implementation of the finite element method in problems of deformation of plates with holes and inclusions, Mathem. methods and phys.-mech. Fields, 56(2) (2013), 48-59.

6. E.L. Hart, V.S. Hudramovich, Projection-iterative schemes for the realization of the finite-element method in problems of deformation of plates with holes and inclusions, J. Math. Sci., 203 (1) (2014), 55-69.

7. E.L. Hart, V.S. Hudramovich, Projection-iterative modification of the method of local variations for problems with a quadratic functional, J. Appl. Math. Mech., 80(2) (2016), 156-163.

8. E.L. HaRT, V.S. Hudramovich, Projection-iterative schemes for the implementation of variational-grid methods in the problems of elastoplastic deformation of inhomogeneous thin-walled structures, J. Math. Sci., 254 (1) (2021), 21-38.

9. E. HART, B. TEROKHIN, Influence of inclusion from functional-gradient material on stress concentration factor in a homogeneous plate with a circular hole, Science and practice: implementation to modern society: Proceedings of the 9th International Scientific and Practical Conference (April 18-19, 2021), Peal Press Ltd, Manchester, Great Britain (2021), 866-872.

10. A. Haque, L. Ahmed, A. Ramasetty, Stress concentrations and notch sensitivity in woven ceramic matrix composites containing a circular hole - an experimental, analytical, and finite element study, J. Amer. Ceramic Soc., 88 (8) (2005), 21952201.

11. V.S. Hudramovich, E.L. Hart, O.A. Marchenko, Reinforcing inclusion effect on the stress concentration within the spherical shell having an elliptical opening under uniform internal pressure, Strength of Materials, 52(6) (2021), 832-842.

12. M. Jabbari, S. Sohrabpour, M.R. Eslami, General solutions for mechanical and thermal stresses in a functionally graded hollow cylinder due to nonaxisymmetric steady-state loads, J. Appl. Mech.-T. ASME, 70 (2003), 111-118.

13. M. JANGHORBAn, A. ZARE, Thermal effect on free vibration analysis of functionally graded arbitrary straight-sided plates with different cutouts, Latin Amer. J. Solids Structures, 8 (2011), 245-257.

14. D.V. Kubair, B. Bhanu-Chandar, Stress concentration factor due to a circular hole in functionally graded panels under uniaxial tension, Intern. J. Mech. Sci., 50 (2008), 732-742.

15. A. Linkov, L. Rybarska-Rusinek, Evaluation of stress concentration in multiwedge systems with functionally graded wedges, Intern. J. Engng Sci., 61 (2012), 87-93.

16. A.J. Markworth, K.S. Ramesh, W.P. Parks, Modelling studies applied to functionally graded materials, J. Mater. Sci., 30 (1995), 2183-2193.

17. Y. Miyamoto, W.A. Kaysser, R.H. Rabin, A. Kawasaki, R.G. Ford, Functionally graded materials: design processing and applications, Kluwer Academic Publishers, USA, 1999.

18. M. Mohammadi, J.R. Dryden, L. Jiang, Stress concentration around a hole in a radially inhomogeneous plate, Intern. J. Solids Structures, 48 (2011), 483-491. 
19. G.N. SAVIN, Stress distribution around holes, Naukova dumka, Kiev, 1968.

20. G.N. SAVIn, V.I. TulchiY, Plates reinforced with compound rings and elastic overlays, Naukova dumka, Kiev, 1971.

21. A. Siasiev, A. Dreus, S. Horbonos, I. Balanenko, S. Dziuba, The stressed-strained state of a rod at crystallization considering the mutual influence of temperature and mechanical fields, Eastern-European Journal of Enterprise Technologies, 3/5(105) (2020), 38-49.

22. A. SyASEv, T. ZelenskAYA, Lengthwise movement dynamic boundary-value problem for trailing boundary ropes, Metallurgical and Mining Industry, 3 (2015), 283287.

23. S.P. Timoshenko, J.N. Goodier, Theory of elasticity, Nauka, Moscow, 1975.

24. K. WAShizU, Variational methods in elasticity and plasticity, Mir, Moscow, 1987.

25. Q. YAnG, C.-F. GAO, W. Chen, Stress analysis of a functional graded material plate with a circular hole, Arch. Appl. Mech., 80 (2010), 895-907.

26. Z. YANG, C.B. KIM, C. CHO, H.G. BEOM, The concentration of stress and strain in finite thickness elastic plate containing a circular hole, Intern. J. Solids Structures, 45 (2008), 713-731.

27. O.C. ZiEnkiewicz, R.L. TEYLOR, The finite element method for solid and structural mechanics, Elsevier, New York, 2005. 